\title{
Infrared Detection of Abundant CS in the Hot Core AFGL 2591 at High Spectral Resolution with SOFIA/EXES*
}

\author{
Andrew G. Barr ${ }^{1}\left(\mathbb{D}\right.$, Adwin Boogert ${ }^{2,8}$, Curtis N. DeWitt ${ }^{3}$, Edward Montiel $^{3}$, Matthew J. Richter ${ }^{3}\left(\mathbb{D}\right.$, Nick Indriolo $^{4}(\mathbb{D}$, \\ David A. Neufeld ${ }^{5}(1)$, Yvonne Pendleton ${ }^{6}$, Jean Chiar $^{7}$, Ryan Dungee ${ }^{2}(1)$, and Alexander G. G. M. Tielens ${ }^{1}$ \\ ${ }_{1}^{1}$ Leiden University Niels Bohrweg 2, 2333 CA Leiden, The Netherlands; barr@strw.leidenuniv.nl \\ ${ }^{2}$ Institute for Astronomy University of Hawaii, 2680 Woodlawn Drive, Honolulu, HI 96822, USA \\ ${ }^{3}$ University of California, Davis, Phys 539, Davis, CA 95616, USA \\ ${ }^{4}$ Space Telescope Science Institute, Baltimore, MD 21218, USA \\ 5 Johns Hopkins University, Baltimore, MD 21218, USA \\ ${ }^{6}$ NASA Ames Research Center, Moffett Field, CA 94035, USA \\ ${ }^{7}$ Diablo Valley College, 321 Golf Club Road, Pleasant Hill, CA 94523, USA \\ Received 2018 August 30; revised 2018 October 19; accepted 2018 October 24; published 2018 November 12
}

\begin{abstract}
We have performed a 5-8 $\mu \mathrm{m}$ spectral line survey of the hot molecular core associated with the massive protostar AFGL 2591, using the Echelon-Cross-Echelle Spectrograph (EXES) on board the Stratospheric Observatory for Infrared Astronomy (SOFIA). We have supplemented these data with a ground-based study in the atmospheric $M$ band around $4.5 \mu \mathrm{m}$ using the iSHELL instrument on the Infrared Telescope Facility (IRTF), and the full $N$-band window from 8 to $13 \mu \mathrm{m}$ using the Texas Echelon Cross Echelle Spectrograph (TEXES) on the IRTF. Here we present the first detection of rovibrational transitions of CS in this source. The absorption lines are centered on average around $-10 \mathrm{~km} \mathrm{~s}^{-1}$ and the line widths of CS compare well with the hot component of ${ }^{13} \mathrm{CO}$ (around $10 \mathrm{~km} \mathrm{~s}^{-1}$ ). Temperatures for $\mathrm{CS}$, hot ${ }^{13} \mathrm{CO}$, and ${ }^{12} \mathrm{CO} v=1-2$ agree well and are around $700 \mathrm{~K}$. We derive a CS abundance of $8 \times 10^{-3}$ and $2 \times 10^{-6}$ with respect to $\mathrm{CO}$ and $\mathrm{H}_{2}$, respectively. This enhanced $\mathrm{CS}$ abundance with respect to the surrounding cloud $\left(1 \times 10^{-8}\right)$ may reflect sublimation of $\mathrm{H}_{2} \mathrm{~S}$ ice followed by gas-phase reactions to form CS. Transitions are in local thermodynamic equilibrium and we derive a density of $>10^{7} \mathrm{~cm}^{-3}$, which corresponds to an absorbing region of $<0.04^{\prime \prime}$. EXES observations of CS are likely to probe deeply into the hot core, to the base of the outflow. Submillimeter and infrared observations trace different components of the hot core as revealed by the difference in systemic velocities, line widths, and temperatures, as well as the CS abundance.
\end{abstract}

Key words: astrochemistry - infrared: ISM - ISM: abundances - ISM: individual objects (AFGL 2591) - line: identification - line: profiles

\section{Introduction}

Hot cores are objects that are formed during the embedded phase of high-mass star formation. They are compact $(\leqslant 0.1 \mathrm{pc})$ regions of dense molecular gas with temperatures and densities $\geqslant 100 \mathrm{~K}$ and $\geqslant 10^{7} \mathrm{~cm}^{-3}$, respectively (Kurtz et al. 2000). They are thought to be an intermediary stage in the formation of massive stars, which starts with the formation of an infrared dark cloud, which then collapses. Once a protostar is formed, it will heat its surroundings, evaporating ice mantles accrued during the preceding cold, dark cloud phase. The resulting rich organic inventory gives rise to a dense forest of spectral lines in the submillimeter (sub-mm) of species such as methanol, dimethyl ether, methyl formate, and many others (Blake et al. 1987; Plambeck \& Wright 1987; Cesaroni 2005). Regions of warm dense gas with a similarly rich organic inventory have also been observed around low-mass stars, where they been been dubbed hot corinos (Ceccarelli 2008).

The physics and chemistry of the hot core phase of massive star formation has been well studied in the sub-mm regime by the analysis of pure rotational line emission at high spectral resolution (Kurtz et al. 2000; van der Tak 2003; Beuther et al. 2007). However, this is not the case for the mid-infrared

\footnotetext{
* Observations made at the IRTF and SOFIA.

8 Staff Astronomer at the Infrared Telescope Facility, which is operated by the University of Hawaii under contract NNH14CK55B with the National Aeronautics and Space Administration.
}

(MIR) spectral range, which contains many strong rovibrational transitions of important molecules, such as $\mathrm{CH}_{4}$ and $\mathrm{C}_{2} \mathrm{H}_{2}$, which do not have transitions in the sub-mm (Lacy et al. 1991).

High-resolution infrared (IR) studies have been carried out before by Mitchell et al. (1990) to analyze CO absorption in a sample of hot cores, including AFGL 2591. Also, Knez et al. (2013) studied the hot core NGC 7538 IRS 1 with the Texas Echelon Cross Echelle Spectrograph (TEXES; Lacy et al. 2002). They detected CS in absorption; however, only six lines were observed, as well as $\mathrm{HNCO}$ and $\mathrm{CH}_{3}$ for the first time in the IR. Indriolo et al. (2015) studied $\mathrm{H}_{2} \mathrm{O}$ absorption toward AFGL 2591 with the Echelon-Cross-Echelle Spectrograph (EXES; Richter et al. 2010).

The results presented in this Letter are part of the first unbiased spectral survey of the 4.5-13 $\mu \mathrm{m}$ region of hot cores, at high spectral resolution $(\mathrm{R}=50,000)$. The high-velocity resolution $\left(6 \mathrm{~km} \mathrm{~s}^{-1}\right)$ allows level specific column densities to be determined, and dynamics studied, for the molecules that are detected. This kind of method has come into reach with instruments such as TEXES and iSHELL (Rayner et al. 2016) on the Infrared Telescope Facility (IRTF), and EXES on board the Stratospheric Observatory for Infrared Astronomy (SOFIA; Young et al. 2012).

In this Letter we present the first detection of rovibrational transitions of CS in AFGL 2591 at MIR wavelengths. CS is well studied in hot cores at sub-mm wavelengths (van der Tak et al. 2003; Tercero et al. 2010; Li et al. 2015). Sulfur-bearing molecules are very sensitive to the physical conditions in hot 
cores and therefore provide good tracers for hot core evolution (Hatchell et al. 2009). Sulfur is also known to be heavily depleted in dense regions (Tieftrunk et al. 1994), and large discrepancies exist between abundances derived from IR and sub-mm observations (Keane et al. 2001; van der Tak et al. 2003). CS, therefore, provides a good candidate for investigating these issues.

\section{Observations and Data Reduction}

AFGL 2591 (R.A. = 20:29:24.80, decl. $=+40: 11: 19.0 ;$ J2000) was observed with the EXES spectrometer on the SOFIA flying observatory as part of program 05_0041 using the highresolution echelon with the low-resolution cross disperser (in order 4). Two settings were observed to cover the wavelength range $(7.57-7.82 \mu \mathrm{m})$ containing the absorption lines of CS reported here. The slit length and width were $3 . ! 2$ and $2 . " 89$, respectively. The spectral resolving power was $R=55,000$, and the sampling was 16 points per resolution element. The observations were done on flights with mission identifications 2017-03-17_EX_F388 and 2017-03-23_EX_F391 at UT 09:51-10:51, latitudes of $45^{\circ}$, longitudes of $-104^{\circ}$, altitudes of $42,000-44,000$ feet, and airmasses of 1.70-1.81. In order to remove background emission, the telescope was nodded between the target position and a position $11^{\prime \prime}$ to the west and $10^{\prime \prime}$ north.

The EXES data were reduced with the SOFIA Redux pipeline (Clarke et al. 2015), which has incorporated routines originally developed for TEXES (Lacy et al. 2002). The science frames were de-spiked and sequential nod positions subtracted to remove telluric emission lines and telescope/ system thermal emission. An internal blackbody source was observed for flat-fielding and flux calibration, and the data were divided by the flat field and then rectified, aligning the spatial and spectral dimensions. The wavenumber calibration was carried out by first examining the sky emission spectrum. This allowed for an easier match to the ATRAN wavenumbers in order to calibrate the dispersion and wavenumber zeropoint. There are two wavenumber solutions as the two settings were taken on different days, and they are accurate to 0.42 and $0.89 \mathrm{~km} \mathrm{~s}^{-1}$. The spectral orders were not flat as a result of instrument fringes. To correct for this, the spectrum was divided over a 201 pixel median-smoothed version of itself. Telluric lines were corrected for using an ATRAN model that was not scaled to the depth of the observed telluric lines. The final signal-to-noise values are 130 and 157 at the native sampling.

AFGL 2591 was observed with iSHELL at the IRTF telescope on Maunakea on UT 2017-07-05 from 13:40 to 15:22 at an airmass range of 1.16-1.50, during good weather conditions as part of program 2017A985. The 0"!375 slit provided a spectral resolving power of 80,000 . The combination of the M1 and M2 configurations provide full coverage from 4.51 to $5.24 \mu \mathrm{m}$. The target was nodded along the $15^{\prime \prime}$ long slit to be able to subtract background emission from the sky and hardware. The total on-target time was 20 minutes for M1 and 31 minutes for M2. iSHELL's internal lamp was used to obtain flat-field images. The spectra were reduced with the Spextool package version 5.0.1 (Cushing et al. 2004). Correction for telluric absorption lines was not done with a standard star but with the program xtellcor_model, ${ }^{9}$ which is newly developed at the IRTF and makes use of the atmospheric

\footnotetext{
9 http://irtfweb.ifa.hawaii.edu/research/dr_resources /
}

models calculated by the Planetary Spectrum Generator (Villanueva et al. 2018). The Doppler shift of AFGL 2591 at the time of the observations was $-35 \mathrm{~km} \mathrm{~s}^{-1}$, and thus telluric and target $\mathrm{CO}$ lines are well separated. The blaze shape of the echelle orders were corrected using the flat fields. The final signal-to-noise is 200 at the native sampling of 2 pixels per resolution element.

\section{Results \\ 3.1. $C S$ and $C O$}

$\mathrm{CS}$ is a linear molecule and, like $\mathrm{CO}$, it has a simple rovibrational spectrum with approximately equidistant lines separated by $2.0 \mathrm{~cm}^{-1}$. We have detected $18 \mathrm{CS}$ absorption lines with excitation energies ranging from 14 to $1317 \mathrm{~K}$. For ${ }^{13} \mathrm{CO}, 16$ lines were detected with an energy range of 5-719 K and 8 lines of the ${ }^{12} \mathrm{CO} v=1-2$ band are detected spanning an energy range of 3200-4234 K. Missing transitions coincide with strong telluric absorption lines or are blended with other hot core lines.

Figure 1 compares molecular line profiles between the sub$\mathrm{mm}$ and IR in AFGL 2591. The CS lines reveal the presence of a single component at approximately $-10 \mathrm{~km} \mathrm{~s}^{-1}$. We have fitted those observed lines with a Gaussian line profile, leaving the width, peak position, and integrated strength as free parameters.

The ${ }^{12} \mathrm{CO} v=0-1$ rovibrational lines are saturated up until $J=9$, and thus we have elected to focus here on the optically thin ${ }^{13} \mathrm{CO}$ and ${ }^{12} \mathrm{CO} v=1-2$ transitions. All components in the ${ }^{12} \mathrm{CO} v=0-1$ line blend into one saturated line except for a broad high-velocity component indicative of a high-velocity outflow. ${ }^{12} \mathrm{CO} v=1-2$ line profiles also show a single component at $-10 \mathrm{~km} \mathrm{~s}^{-1}$. The line parameters derived from the Gaussian fits are summarized in Table 1. The line widths have been de-convolved with the instrumental resolution.

The ${ }^{13} \mathrm{CO}$ lines show complex profiles with multiple velocity components (Figure 1), one of which coincides in velocity with the CS lines and ${ }^{12} \mathrm{CO} v=1-2$ transitions. Two temperature components (hot and cold; see Figure 2) are observed at this velocity. For the hot component (high $J$ ), we adopted the CS line width, but the line width for the cold component (low J) had to be reduced (to $4.1 \mathrm{~km} \mathrm{~s}^{-1}$ ). Absorption by the narrower cold component overwhelms the contribution by the warm component at the lower $J$-levels.

The detected, unblended lines of CS and CO are presented in Figure 3 in the Appendix. There is a spread in centroid velocity for a given species. The line widths for $\mathrm{CS}$ and hot ${ }^{13} \mathrm{CO}$ are in agreement for equivalent energy level.

The rotation diagrams of $\mathrm{CS},{ }^{13} \mathrm{CO}$, and ${ }^{12} \mathrm{CO} v=1-2$ are shown in Figure 2. In all cases the rotation diagrams are straight lines, indicating that local thermodynamic equilibrium (LTE) is a good approximation. We have calculated the vibrational excitation temperature of ${ }^{12} \mathrm{CO}$ from the $v=1-2$ transitions and the warm ${ }^{13} \mathrm{CO}$ column density assuming a ${ }^{12} \mathrm{CO} /{ }^{13} \mathrm{CO}$ abundance ratio of 60 (Wilson \& Rood 1994). The derived vibrational excitation temperature of $625 \pm 56 \mathrm{~K}$ agrees well with a similar estimate by Mitchell et al. (1989). The similar values for the rotational and vibrational temperatures suggest vibrational LTE.

The excitation temperature and velocity for all three species $\left(\mathrm{CS},{ }^{13} \mathrm{CO}\right.$ high $J$, and $\left.{ }^{12} \mathrm{CO} v=1-2\right)$ are very similar. This supports an origin in the same region. The physical conditions 

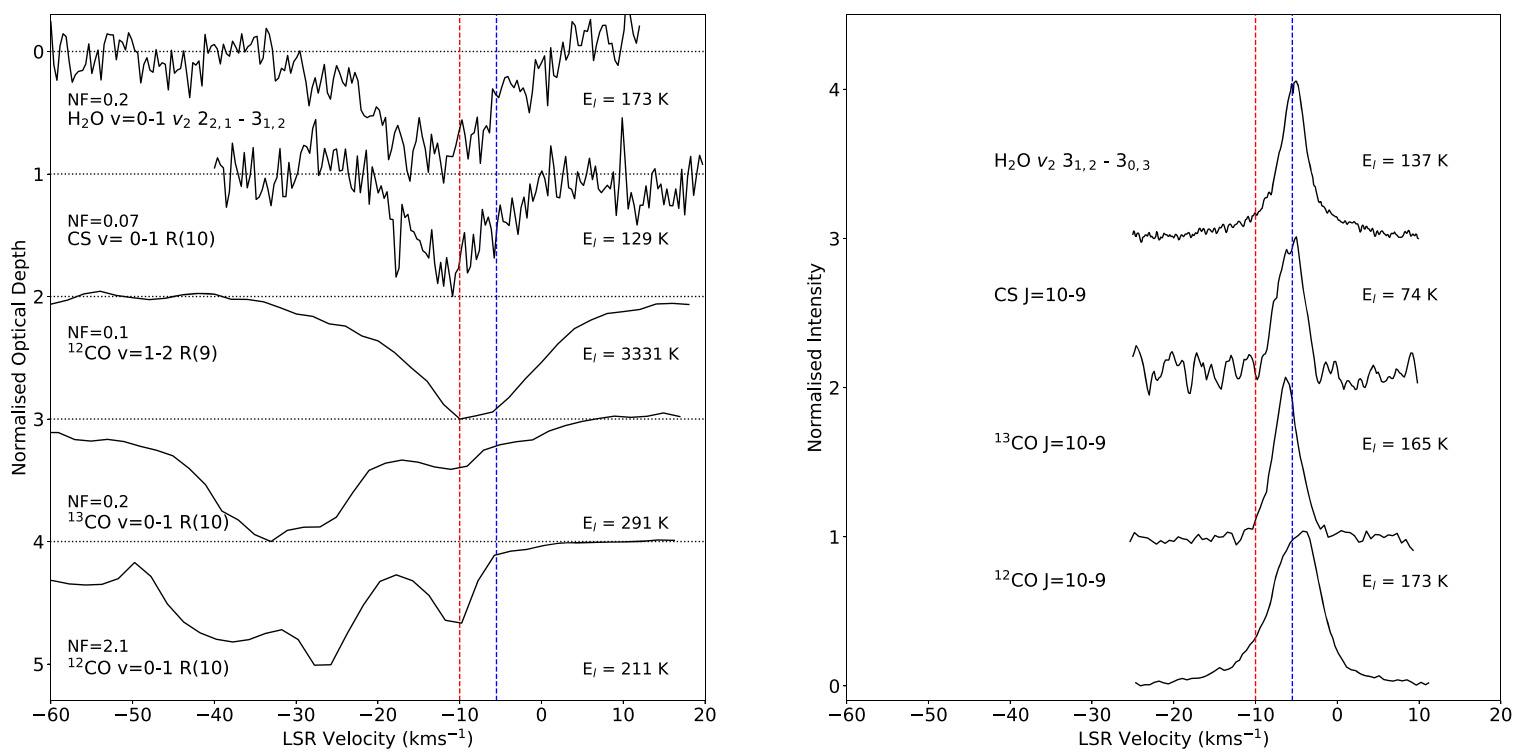

Figure 1. Comparison between IR and sub-mm line profiles. The IR lines are shown as optical depth profiles. The sub-mm profiles for CS, ${ }^{13} \mathrm{CO}$, and ${ }^{12} \mathrm{CO}$ are taken from Kaźmierczak-Barthel et al. (2014) and the sub- $\mathrm{mm} \mathrm{H}_{2} \mathrm{O}$ line is taken from Choi et al. (2015). The IR $\mathrm{H}_{2} \mathrm{O}$ line is from our EXES data. The dashed red and blue lines indicate $-10 \mathrm{~km} \mathrm{~s}^{-1}$ and $-5.5 \mathrm{~km} \mathrm{~s}^{-1}$, respectively. Lines are normalized and lower energy levels given. For the IR lines, normalization factors (NF) are given and horizontal baselines are shown.

for the detected species are summarized in Table 2. An estimate of the column density of hot ${ }^{12} \mathrm{CO}$ from the lines of vibrationally excited ${ }^{12} \mathrm{CO}$ gives $1.5 \pm 0.6 \times 10^{18} \mathrm{~cm}^{-2}$. We derive an upper limit on $\mathrm{CS}$ in the $-33 \mathrm{~km} \mathrm{~s}^{-1}$ velocity component of $<8 \times 10^{14} \mathrm{~cm}^{-2}$.

\subsection{CS and CO: Infrared versus Submillimeter}

From our observations we derive a different systemic velocity than from sub-mm studies of AFGL 2591 (Figure 1) in $\mathrm{CS},{ }^{13} \mathrm{CO}$, and ${ }^{12} \mathrm{CO} v=1-2\left(-10 \mathrm{~km} \mathrm{~s}^{-1}\right.$ compared to $-5.5 \mathrm{~km} \mathrm{~s}^{-1}$; Bally \& Lada 1983). van der Tak et al. (1999) studied AFGL 2591 at sub-mm, radio, and IR wavelengths, at lower resolution, and derived a centroid velocity for ${ }^{13} \mathrm{CO}$ absorption of $-5.5 \mathrm{~km} \mathrm{~s}^{-1}$, in agreement with the systemic velocity of rotational emission lines, including CS (Boonman et al. 2001; Benz et al. 2007; Kaźmierczak-Barthel et al. 2014). However, their IR transitions show a spread in velocity of -3.5 to $-12.4 \mathrm{~km} \mathrm{~s}^{-1}$ which they attribute to atmospheric interference, picking only a few lines to derive their value of $-5.5 \mathrm{~km} \mathrm{~s}^{-1}$.

The intrinsic line widths that we observed are broader than for sub-mm studies (Figure 1). As an example, the CS $J=10-9$ transition has a width of $3.5 \mathrm{~km} \mathrm{~s}^{-1}$, whereas our CS rovibrational transition $v=0-1 \mathrm{R}(10)$ has a width of $10.4 \mathrm{~km} \mathrm{~s}^{-1}$. The $\mathrm{H}_{2} \mathrm{O}$ $3(1,2)-3(0,3)$ line has a width of $3.1 \mathrm{~km} \mathrm{~s}^{-1}$ in the sub-mm compared to $16.5 \mathrm{~km} \mathrm{~s}^{-1}$ for the $\nu_{2}=0-12(2,1)-3(1,2)$ IR line.

We suggest that our higher-temperature, higher-velocity IR observations trace more turbulent gas closer to the central source, probing deep into the base of the outflow, whereas sub$\mathrm{mm}$ observations trace more extended gas at the velocity of the quiescent envelope.

The CS, ${ }^{13} \mathrm{CO} v=0-1$, and ${ }^{12} \mathrm{CO} v=1-2$ temperatures are very similar and, given the difference in critical densities, suggest gas in LTE. The critical densities for $J=7-6$ are $2 \times 10^{5} \mathrm{~cm}^{-3}$ (Yang et al. 2010) and $3 \times 10^{7} \mathrm{~cm}^{-3}$ (Turner et al. 1992) for CO and CS, respectively. Therefore, we estimate that the density must exceed $10^{7} \mathrm{~cm}^{-3}$ in order to maintain LTE. The critical density of ${ }^{12} \mathrm{CO} v=1-2$ cannot be used due to the high optical depth of the ${ }^{12} \mathrm{CO} v=0-1$ transitions, which may lead to line trapping and tends to increase the vibrational excitation temperature.

Assuming a ${ }^{12} \mathrm{CO} / \mathrm{H}_{2}$ abundance of $2 \times 10^{-4}$ (Lacy et al. 1994) and constant density of $10^{7} \mathrm{~cm}^{-3}$, we derive a physical size for the absorbing region of $<130 \mathrm{au}$. At a distance of $3.3 \mathrm{kpc}$ (Rygl et al. 2012) this corresponds to $<0.04^{\prime \prime}$.

The velocity structure of the inner region (down to $500 \mathrm{au}$ ) of the hot core in AFGL 2951 has been well resolved and modeled by Wang et al. (2012). A high-velocity gradient is found for $\mathrm{SO}_{2}$ and the velocity field of the blueshifted component is observed to have the highest negative velocities toward the center on the source. However, the highest velocity that is reached is $\sim-7 \mathrm{~km} \mathrm{~s}^{-1}$, at the very center. This further indicates that CS at $-10 \mathrm{~km} \mathrm{~s}^{-1}$ would not be observed at sub$\mathrm{mm}$ wavelengths. Combined with the fact that $\mathrm{SO}_{2}$ is a molecular tracer for shocks or outflows (Schilke et al. 1997), Wang et al. (2012) concluded that the $\mathrm{SO}_{2}$ emission originates in an interactive layer between the upper parts of a disk-like structure and the outflow.

van der Tak et al. (1999) concluded that the outflow of AFGL 2591 points toward us along the line of sight. In order to explain CS (7-6) emission, Bruderer et al. (2009) proposed that their observations trace dense walls of the outflow, at a velocity of around $-5.5 \mathrm{~km} \mathrm{~s}^{-1}$. Given that the extreme velocities originate in the center of the source, and that our EXES observations show an average velocity around $-10 \mathrm{~km} \mathrm{~s}^{-1}$, we propose that we are looking into the outflow, probing deeply toward the protostar to the base of the outflow. Also the broad line profiles of CS are indicative of a shocked region. The high temperature implies that the gas lies close to the protostar, further supporting the proposed origin as the base of the outflow. 
Table 1

CS and CO Line Parameters

\begin{tabular}{|c|c|c|c|c|c|c|c|c|c|}
\hline Species & Transition & Wavelength $(\mu \mathrm{m})$ & $E_{l}(\mathrm{~K})$ & $g_{l}$ & $A_{i j}\left(\mathrm{~s}^{-1}\right)$ & $v_{\mathrm{lsr}}\left(\mathrm{km} \mathrm{s}^{-1}\right)$ & $\Delta v\left(\mathrm{~km} \mathrm{~s}^{-1}\right)$ & $\tau_{0}$ & $N_{l}\left(\times 10^{14} \mathrm{~cm}^{-2}\right)$ \\
\hline \multirow[t]{18}{*}{$\mathrm{CS} v=0-1$} & $\mathrm{R}(3)$ & 7.8212 & 14.1 & 7 & 7.2 & $-11.9 \pm 0.5$ & $8.3 \pm 1.5$ & $0.031 \pm 0.004$ & $1.9 \pm 0.4$ \\
\hline & $\mathrm{R}(4)^{\mathrm{a}}$ & 7.8116 & 23.5 & 9 & 7.4 & $-9.4 \pm 0.5$ & $6.1 \pm 1.3$ & $0.032 \pm 0.004$ & $1.6 \pm 0.4$ \\
\hline & $\mathrm{R}(5)$ & 7.8020 & 35.3 & 11 & 7.6 & $-10.1 \pm 0.5$ & $7.7 \pm 1.3$ & $0.039 \pm 0.004$ & $2.3 \pm 0.5$ \\
\hline & $\mathrm{R}(7)^{\mathrm{a}}$ & 7.7833 & 65.8 & 15 & 7.8 & $-10.4 \pm 0.2$ & $6.4 \pm 0.6$ & $0.056 \pm 0.003$ & $3.0 \pm 0.3$ \\
\hline & $\mathrm{P}(8)$ & 7.9443 & 84.6 & 17 & 8.1 & $-10.9 \pm 0.5$ & $9.6 \pm 1.5$ & $0.055 \pm 0.006$ & $4.5 \pm 0.9$ \\
\hline & $\mathrm{R}(9)$ & 7.7649 & 105.8 & 19 & 8.0 & $-9.5 \pm 0.6$ & $11.2 \pm 1.8$ & $0.051 \pm 0.005$ & $4.1 \pm 0.5$ \\
\hline & $\mathrm{R}(10)$ & 7.7559 & 129.2 & 21 & 8.0 & $-11.1 \pm 0.3$ & $10.4 \pm 0.9$ & $0.061 \pm 0.004$ & $4.7 \pm 0.5$ \\
\hline & $\mathrm{R}(11)$ & 7.7469 & 155.1 & 23 & 8.1 & $-12.1 \pm 0.3$ & $9.9 \pm 0.8$ & $0.060 \pm 0.002$ & $4.4 \pm 0.4$ \\
\hline & $\mathrm{R}(18)$ & 7.6868 & 401.8 & 37 & 8.5 & $-12.0 \pm 0.3$ & $11.0 \pm 0.8$ & $0.063 \pm 0.003$ & $5.0 \pm 0.6$ \\
\hline & $\mathrm{R}(22)^{\mathrm{a}}$ & 7.6545 & 594.5 & 45 & 8.7 & $-12.7 \pm 0.4$ & $10.7 \pm 1.2$ & $0.080 \pm 0.004$ & $6.1 \pm 2.4$ \\
\hline & $\mathrm{R}(23)$ & 7.6467 & 648.5 & 47 & 8.8 & $-10.3 \pm 0.9$ & $8.4 \pm 3.8$ & $0.016 \pm 0.003$ & $4.2 \pm 1.1$ \\
\hline & $\mathrm{R}(24)^{\mathrm{a}}$ & 7.6389 & 704.7 & 49 & 8.8 & $-5.2 \pm 0.7$ & $15.0 \pm 1.8$ & $0.060 \pm 0.003$ & $5.9 \pm 1.9$ \\
\hline & $\mathrm{R}(26)$ & 7.6237 & 824.4 & 53 & 8.9 & $-8.4 \pm 0.6$ & $7.9 \pm 2.0$ & $0.048 \pm 0.007$ & $3.0 \pm 0.7$ \\
\hline & $\mathrm{R}(27)^{\mathrm{a}}$ & 7.6162 & 887.8 & 55 & 9.0 & $-11.2 \pm 0.6$ & $4.9 \pm 1.2$ & $0.076 \pm 0.006$ & $3.6 \pm 0.8$ \\
\hline & $\mathrm{R}(28)$ & 7.6088 & 953.4 & 57 & 9.0 & $-10.0 \pm 0.4$ & $6.8 \pm 1.0$ & $0.055 \pm 0.008$ & $3.1 \pm 0.7$ \\
\hline & $\mathrm{R}(29)$ & 7.6015 & 1021.5 & 59 & 9.1 & $-10.9 \pm 0.4$ & $6.7 \pm 1.2$ & $0.078 \pm 0.008$ & $4.4 \pm 0.8$ \\
\hline & $\mathrm{R}(31)^{\mathrm{a}}$ & 7.5871 & 1164.5 & 63 & 9.2 & $-8.0 \pm 0.9$ & $11.4 \pm 4.1$ & $0.024 \pm 0.005$ & $1.8 \pm 0.8$ \\
\hline & $\mathrm{R}(33)$ & 7.5731 & 1316.8 & 67 & 9.2 & $-9.0 \pm 0.6$ & $5.2 \pm 1.5$ & $0.051 \pm 0.009$ & $2.5 \pm 0.7$ \\
\hline \multirow[t]{16}{*}{${ }^{13} \mathrm{CO} v=0-1$} & $\mathrm{P}(1)$ & 4.7792 & 5.2 & 6 & 32.4 & $-9.4 \pm 0.2$ & 4.1 & $0.50 \pm 0.04$ & $72.4 \pm 7.3$ \\
\hline & $\mathrm{P}(2)$ & 4.7877 & 15.8 & 10 & 21.5 & $-9.3 \pm 0.1$ & 4.1 & $0.57 \pm 0.02$ & $77.5 \pm 3.6$ \\
\hline & $\mathrm{R}(2)$ & 4.7463 & 15.8 & 10 & 14.2 & $-9.7 \pm 0.3$ & 4.1 & $0.68 \pm 0.07$ & $67.0 \pm 8.0$ \\
\hline & $\mathrm{R}(3)$ & 4.7383 & 31.7 & 14 & 14.8 & $-9.3 \pm 0.1$ & 4.1 & $0.70 \pm 0.02$ & $68.6 \pm 2.7$ \\
\hline & $\mathrm{P}(3)$ & 4.7963 & 31.7 & 14 & 19.3 & $-9.3 \pm 0.2$ & 4.1 & $0.61 \pm 0.04$ & $79.0 \pm 6.2$ \\
\hline & $\mathrm{P}(4)$ & 4.8050 & 52.8 & 18 & 18.2 & $-9.3 \pm 0.2$ & 4.1 & $0.57 \pm 0.03$ & $72.4 \pm 5.2$ \\
\hline & $\mathrm{R}(5)$ & 4.7227 & 79.3 & 22 & 15.5 & $-8.3 \pm 0.2$ & 4.1 & $0.44 \pm 0.06$ & $38.6 \pm 5.7$ \\
\hline & $\mathrm{R}(6)$ & 4.7150 & 111.0 & 26 & 15.8 & $-9.1 \pm 0.3$ & 4.1 & $0.31 \pm 0.06$ & $29.3 \pm 5.9$ \\
\hline & $\mathrm{R}(9)$ & 4.6927 & 237.9 & 38 & 16.4 & $-12.1 \pm 0.7$ & 11.2 & $0.14 \pm 0.01$ & $23.6 \pm 3.1$ \\
\hline & $\mathrm{P}(9)$ & 4.8501 & 237.9 & 38 & 16.4 & $-13.2 \pm 0.8$ & 11.2 & $0.10 \pm 0.01$ & $18.3 \pm 2.6$ \\
\hline & $\mathrm{R}(10)$ & 4.6853 & 290.8 & 42 & 16.5 & $-10.5 \pm 0.6$ & 11.2 & $0.10 \pm 0.01$ & $17.5 \pm 2.5$ \\
\hline & $\mathrm{R}(11)$ & 4.6782 & 349.0 & 46 & 16.7 & $-12.0 \pm 0.5$ & 11.2 & $0.11 \pm 0.01$ & $18.9 \pm 2.3$ \\
\hline & $\mathrm{P}(11)$ & 4.8689 & 349.0 & 46 & 16.1 & $-13.0 \pm 0.6$ & 11.2 & $0.05 \pm 0.01$ & $10.0 \pm 1.8$ \\
\hline & $\mathrm{R}(12)$ & 4.6711 & 412.3 & 50 & 16.8 & $-12.0 \pm 0.6$ & 11.2 & $0.09 \pm 0.01$ & $16.9 \pm 1.9$ \\
\hline & $\mathrm{R}(13)$ & 4.6641 & 481.1 & 54 & 16.9 & $-12.0 \pm 0.4$ & 11.2 & $0.09 \pm 0.01$ & $15.9 \pm 1.4$ \\
\hline & $R(16)$ & 4.6437 & 718.8 & 66 & 17.3 & $-10.8 \pm 0.5$ & 11.2 & $0.10 \pm 0.01$ & $16.6 \pm 1.8$ \\
\hline \multirow[t]{8}{*}{${ }^{12} \mathrm{CO} v=1-2$} & $\mathrm{R}(6)$ & 4.6675 & 3199.5 & 13 & 33.2 & $-9.6 \pm 0.6$ & $17.9 \pm 1.4$ & $0.102 \pm 0.006$ & $13.1 \pm 1.6$ \\
\hline & $\mathrm{R}(7)$ & 4.6598 & 3237.9 & 15 & 33.7 & $-8.2 \pm 0.7$ & $19.9 \pm 1.9$ & $0.108 \pm 0.008$ & $15.6 \pm 2.6$ \\
\hline & $\mathrm{R}(8)$ & 4.6522 & 3281.8 & 17 & 34.1 & $-9.1 \pm 0.4$ & $18.3 \pm 0.9$ & $0.124 \pm 0.006$ & $16.4 \pm 1.4$ \\
\hline & $\mathrm{R}(9)$ & 4.6448 & 3331.0 & 19 & 34.4 & $-8.6 \pm 0.4$ & $17.9 \pm 0.9$ & $0.113 \pm 0.005$ & $14.8 \pm 1.2$ \\
\hline & $\mathrm{R}(17)$ & 4.5887 & 3922.5 & 35 & 36.6 & $-8.5 \pm 0.3$ & $16.6 \pm 0.9$ & $0.104 \pm 0.005$ & $12.7 \pm 1.1$ \\
\hline & $\mathrm{P}(18)$ & 4.8948 & 4020.9 & 37 & 31.7 & $-7.1 \pm 0.3$ & $13.9 \pm 0.9$ & $0.083 \pm 0.004$ & $9.4 \pm 0.9$ \\
\hline & $\mathrm{P}(19)$ & 4.9054 & 4124.8 & 39 & 31.4 & $-9.9 \pm 0.8$ & $14.6 \pm 3.8$ & $0.010 \pm 0.009$ & $12.0 \pm 1.9$ \\
\hline & $\mathrm{R}(20)$ & 4.5694 & 4234.4 & 41 & 37.2 & $-6.1 \pm 0.5$ & $16.0 \pm 1.2$ & $0.088 \pm 0.005$ & $10.3 \pm 1.5$ \\
\hline
\end{tabular}

Notes. $\Delta v$ is the line FWHM, $\tau_{0}$ is the optical depth at line center, and $N_{l}$ is the column density in the lower energy level of the transition, with energy $E_{l} . A_{i j}$ is the Einstein $A$ coefficient for the transition and $g_{l}$ is the statical weight.

${ }^{a}$ These lines suffer from systematic errors such as poor baselines, therefore there is a larger uncertainty in the continuum placement. Line data were taken from the high-resolution transmission molecular absorption (HITRAN) database (Gordon et al. 2017).

\subsection{Chemistry of $C S$}

We derive a CS $/{ }^{12} \mathrm{CO}$ abundance of $8 \times 10^{-3}$ and $\mathrm{CS} / \mathrm{H}_{2}$ abundance of $2 \times 10^{-6}$, assuming a $\mathrm{CO} / \mathrm{H}_{2}$ ratio of $2 \times 10^{-4}$. This abundance is two orders of magnitude higher than sub$\mathrm{mm}$ observations of the hot core and envelope (van der Tak et al. 2003; Jiménez-Serra et al. 2012). This, once again, illustrates that the IR observations probe a very different region in this source. Consequently, hot CS contains $6 \pm 0.8 \%$ of the cosmic sulfur budget, assuming an $\mathrm{S} / \mathrm{H}$ ratio of $1.3 \times 10^{-5}$. Compared to the Orion Hot Core, which has a CS/CO abundance of $4.1 \times 10^{-4}$ derived from sub-mm observations (Tercero et al. 2010), the CS abundance in the gas traced by the IR observations of the hot core in AFGL 2591 is much higher.
Chemical models based on sub-mm observations (Charnley 1997; Doty et al. 2002) derive a CS abundance of the order $1 \times 10^{-8}$ with respect to $\mathrm{H}_{2}$. A two phase, time-dependent gasgrain model (Viti et al. 2004) has been used to study the sulfur chemistry in the Orion Hot Core, and predicts the abundance of CS as a function of hot core age (Esplugues et al. 2014). The first and second phase simulate depletion onto, and sublimation from, grain surfaces, respectively. For a hot core with a mass of $10 M_{\odot}$, solar sulfur abundance and a density of $10^{7} \mathrm{~cm}^{-3}$, a $\mathrm{CS} / \mathrm{CO}$ abundance of around $5 \times 10^{-3}$ is achieved after $6 \times 10^{4} \mathrm{yr}$.

The abundances of $\mathrm{CS}, \mathrm{H}_{2} \mathrm{CS}$, and $\mathrm{SO}_{2}$ increase such that these species become the most abundant sulfur-bearing molecules for an evolved hot core (Esplugues et al. 2014). 

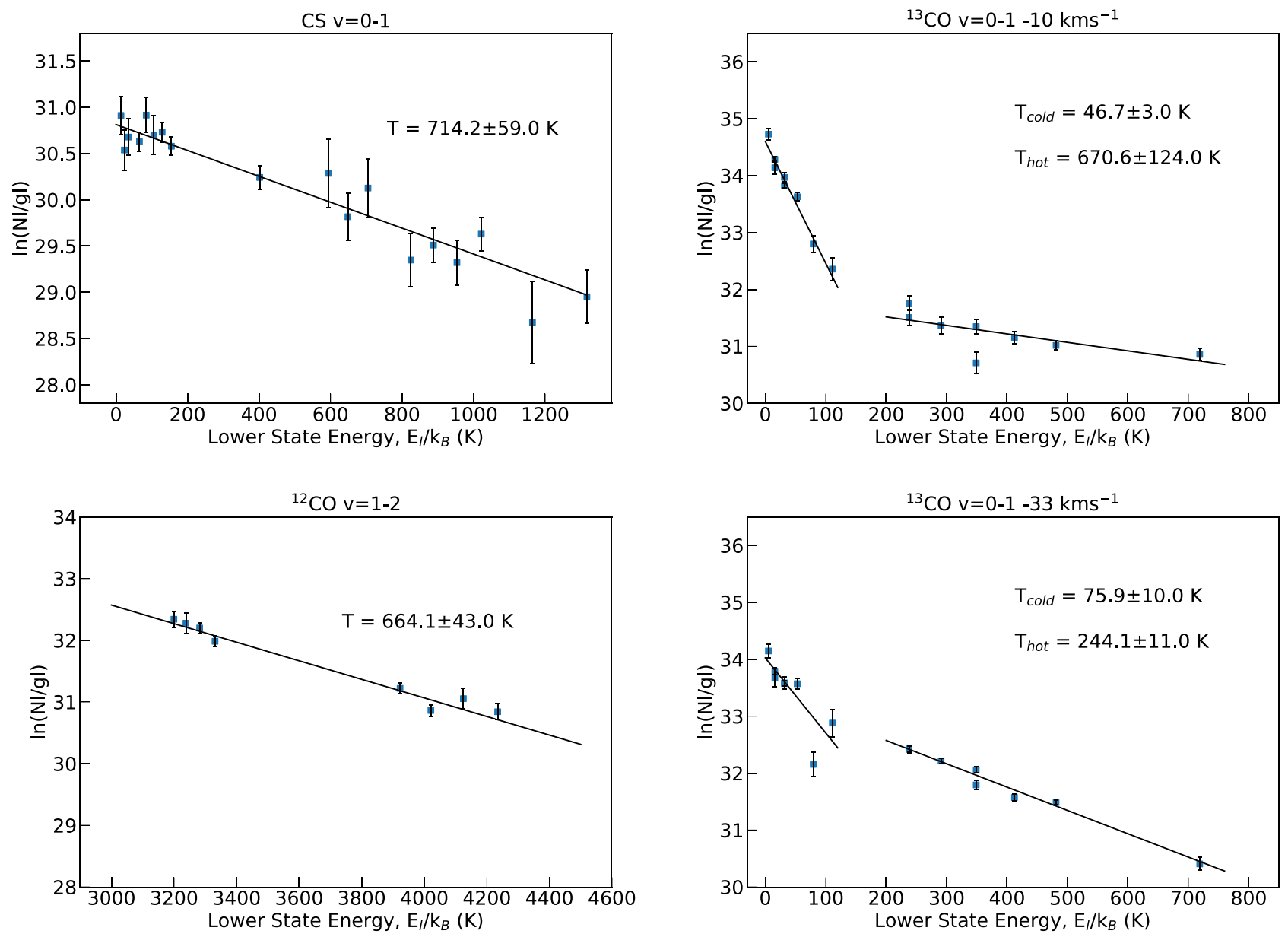

Figure 2. CS and CO rotation diagrams. Temperatures for each species are shown, with two temperature components for ${ }^{13} \mathrm{CO}$.

The main chemical pathway that is responsible for the production of $\mathrm{CS}$ in this model is $\mathrm{CH}_{2}+\mathrm{S} \rightarrow \mathrm{CS}+\mathrm{H}_{2}$. Chemical models predict large amounts of atomic sulfur at high temperatures (Doty et al. 2002). This arises due to abstraction of $\mathrm{H}_{2} \mathrm{~S}$, which is efficient at high temperatures. At the same time, small hydrocarbons are formed by the breakdown of $\mathrm{CO}$ via cosmic ray ionization.

Therefore, we propose two scenarios to interpret the CS abundance. The first is that AFGL 2591 is a more evolved hot core in which all sulfur is converted to $\mathrm{H}_{2} \mathrm{~S}$ on grain surfaces, and then converted back to $\mathrm{S}$ in the gas-phase after ice mantle sublimation. Then, at long timescales, enough CS is produced in the hot inner region of the hot core to explain our observations (Esplugues et al. 2014). These models are not optimized to AFGL 2591, which would likely have an effect on the CS abundance, therefore a more in-depth study using chemical models would be necessary to clarify the proposed timescales. We note that $\mathrm{H}_{2} \mathrm{~S}$ ice has not been observed in absorption toward massive protostars (Smith 1991) at abundance upper limits a factor of 7 lower than that of CS, putting $\mathrm{H}_{2} \mathrm{~S}$ as a source of the gas phase $\mathrm{S}$ into question. Deeper searches for $\mathrm{H}_{2} \mathrm{~S}$ would be very helpful to asses the sulfur budget in interstellar ices.

The alternative scenario is that our observations trace a diskwind interaction zone very close to the protostar. In this case, the cosmic ray ionization rate would be high, which would favor the breaking of $\mathrm{C}$ out of $\mathrm{CO}$, which could lead to the enhancement of CS production. Again, atomic sulfur is produced via abstraction of $\mathrm{H}_{2} \mathrm{~S}$. May et al. (2000) found that grain sputtering becomes important in shocks around $15 \mathrm{~km} \mathrm{~s}^{-1}$, therefore sulfur could also be released from grain mantles in the presence of shocks.

The conditions and chemical history of AFGL 2591 are clearly favorable for the production of CS. A high CS/CO abundance of $7 \pm 0.4 \times 10^{-3}$ has also been observed at MIR wavelengths in NGC 7538 IRS 1 (Knez et al. 2013) with TEXES. Nevertheless, the enhanced CS abundance is still not enough to appoint this molecule as the main reservoir of sulfur in hot cores. A high abundance of warm $\mathrm{SO}_{2}$ has also been observed with EXES in the hot core Mon R2 IRS 3. (Dungee et al. 2018). This also is not observed in the sub-mm, suggesting that a large amount of sulfur is visible only at IR wavelengths.

\section{Conclusions}

We present the first detection of rovibrational transitions of CS in the hot core of AFGL 2591 with EXES. The CS observations are complemented with high-resolution iSHELL $\mathrm{CO}$ observations. The $\mathrm{CO}$ gas is found to have five velocity components, one of which is consistent with the velocity of CS, $-10 \mathrm{~km} \mathrm{~s}^{-1}$. ${ }^{12} \mathrm{CO} v=1-2$ is also observed at this velocity. A temperature of $714 \pm 59 \mathrm{~K}$ is derived from the rotation diagram of CS, and the observation of CS up to $J$ level of 33 , along with a similar excitation temperature for the pure rotational CO lines, imply high densities $\left(>10^{7} \mathrm{~cm}^{-3}\right)$. The temperature is consistent with hot ${ }^{13} \mathrm{CO}$ and ${ }^{12} \mathrm{CO} v=1-2$ which have $670 \pm 124 \mathrm{~K}$ and $664 \pm 43 \mathrm{~K}$, respectively. 
Table 2

Summary of Molecular Species in AFGL 2591

\begin{tabular}{|c|c|c|c|c|}
\hline Species & $v_{\mathrm{lsr}}\left(\mathrm{km} \mathrm{s}^{-1}\right)$ & $T_{\mathrm{ex}}(\mathrm{K})$ & $N\left(\times 10^{16} \mathrm{~cm}^{-2}\right)$ & $\bar{N} / N\left({ }^{12} \mathrm{CO}\right)^{\mathrm{a}}$ \\
\hline $\mathrm{CS} v=0-1$ & $-10 \pm 0.5$ & $714 \pm 59$ & $1.6 \pm 0.1$ & $8.0 \times 10^{-3}$ \\
\hline${ }^{12} \mathrm{CO} v=1-2$ & $-8.4 \pm 0.5$ & $664 \pm 43$ & $1.48 \pm 0.6$ & $7.4 \times 10^{-3}$ \\
\hline${ }^{13} \mathrm{CO} v=0-1$ & $-11.95 \pm 0.6$ & $670 \pm 124$ & $3.4 \pm 0.4$ & 0.02 \\
\hline${ }^{13} \mathrm{CO} v=0-1$ & $-9.2 \pm 0.2$ & $47 \pm 3$ & $3.8 \pm 0.2$ & 0.02 \\
\hline${ }^{13} \mathrm{CO} v=0-1$ & $-33 \pm 0.8$ & $76 \pm 10$ & $3.4 \pm 0.2$ & 0.02 \\
\hline${ }^{13} \mathrm{CO} v=0-1$ & $-33 \pm 0.5$ & $244 \pm 11$ & $5.9 \pm 0.4$ & 0.03 \\
\hline
\end{tabular}

Notes.

${ }^{\mathrm{a}} N\left({ }^{12} \mathrm{CO}\right)=N\left({ }^{13} \mathrm{CO}\right) \times 60$ (Wilson \& Rood 1994).

${ }^{\mathrm{b}}$ Indriolo et al. (2015) with EXES.

The systemic velocity of AFGL 2591 that we derive is $5 \mathrm{~km} \mathrm{~s}^{-1}$ bluer than that derived from sub-mm observations. We propose that this is because we are observing the base of the blueshifted outflow very close to the central IR source. This is reflected in the high densities and temperatures derived in our observations.

The abundance of CS observed to be $8 \times 10^{-3}$ and $2 \times 10^{-6}$ with respect to $\mathrm{CO}$ and $\mathrm{H}_{2}$, respectively. This is two orders of magnitude above what is derived from sub-mm observations, $1 \times 10^{-8}$ with respect to $\mathrm{H}_{2}$. This provides evidence of a large sulfur depository which is detectable more readily at IR wavelengths. IR observations are sensitive to a different region of the hot core than sub-mm observations. IR observations of CS trace gas in the hot core that is much hotter and denser than sub$\mathrm{mm}$ observations do, and that is at a larger systemic velocity. Therefore, they probe much deeper into the innermost parts of the hot core, avoiding any contamination by the surrounding envelope.
Chemical models support the derived abundance of CS if AFGL 2591 is an evolved hot core. Alternatively, our observations may be tracing the onset of a disk wind at the base of the outflow.

Based [in part] on observations made with the NASA/DLR Stratospheric Observatory for Infrared Astronomy (SOFIA). SOFIA is jointly operated by the Universities Space Research Association, Inc. (USRA), under NASA contract NAS2-97001, and the Deutsches SOFIA Institut (DSI) under DLR contract 50 OK 0901 to the University of Stuttgart. A.G.G.M.T thanks the Spinoza premie of the NWO. D.A.N gratefully acknowledges the support of an USRA SOFIA grant, SOF05-0041.

\section{Appendix}

Plots of detected, unblended lines of $\mathrm{CS},{ }^{13} \mathrm{CO} v=0-1$, and ${ }^{12} \mathrm{CO} v=1-2$. 

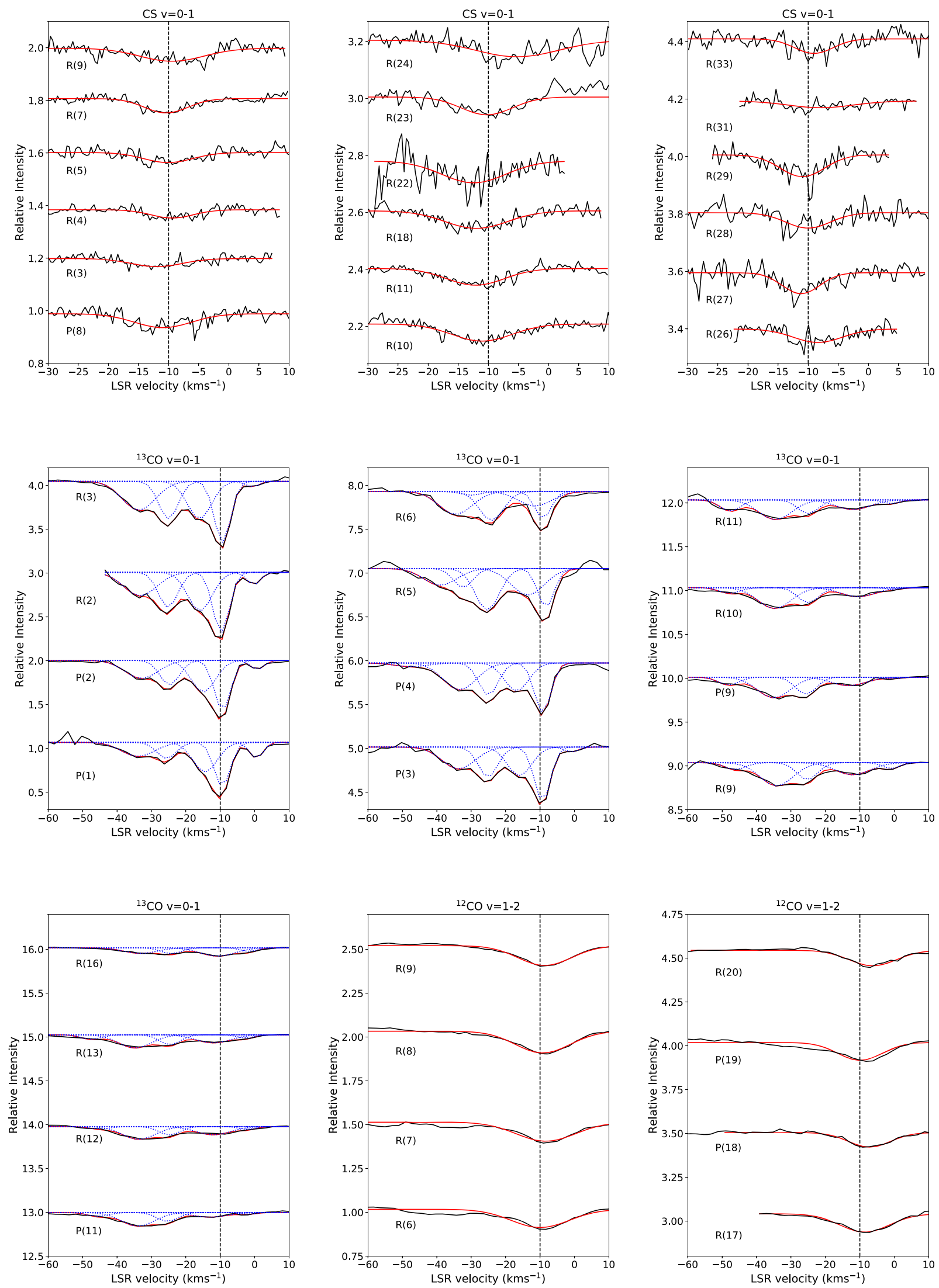

Figure 3. All lines of $\mathrm{CS},{ }^{13} \mathrm{CO}$, and ${ }^{12} \mathrm{CO} v=1-2$. The blue dotted lines represent the individual velocity components, and the red solid lines show the overall fit. The black dashed line denotes $-10 \mathrm{~km} \mathrm{~s}^{-1}$. 


\section{ORCID iDs}

Andrew G. Barr (i) https://orcid.org/0000-0003-4909-2770

Matthew J. Richter (1) https://orcid.org/0000-0002-8594-2122

Nick Indriolo (1) https://orcid.org/0000-0001-8533-6440

David A. Neufeld (i) https://orcid.org/0000-0001-8341-1646

Ryan Dungee iㅣ https://orcid.org/0000-0001-6669-0217

\section{References}

Bally, J., \& Lada, C. J. 1983, ApJ, 265, 824

Benz, A. O., Staüber, P., Bourke, T. L., van der Tak, F. F. S., et al. 2007, A\&A, 475,549

Beuther, H., Churchwell, E. B., McKee, C. F., \& Tan, J. C. 2007, in Protostars and Planets V, ed. B. Reipurth, D. Jewitt, \& K. Keil (Tucson: Univ. Arizona Press), 165

Blake, G. A., Sutton, E. C., Masson, C. R., \& Phillips, T. G. 1987, ApJ, 315, 621

Boonman, A. M. S., Stark, R., van der Tak, F. F. S., et al. 2001, ApJL, 553, L63

Bruderer, S., Benz, A. O., Bourke, T. L., \& Doty, S. D. 2009, A\&A, 503, L13

Carr, J. S., Evans, N. J., Lacy, J. H., \& Zhou, S. 1995, ApJ, 450, 667

Ceccarelli, C. 2008, in IAU Symp. Proc. 251, Organic Matter in Space, ed. S. Kwok \& S. Sandford (Cambridge: Cambridge Univ. Press), 79

Cesaroni, R. 2005, in IAU Symp. 227, Massive Star Birth: A Crossroads of Astrophysics, ed. R. Cesaroni et al. (Cambridge Univ. Press: Cambridge), 59 Charnley, S. B. 1997, ApJ, 481, 396

Choi, Y., van der Tak, F. F. S., van Dishoeck, E. F., Herpin, F., \& Wyrowski, F. 2015, A\&A, 576, A85

Clarke, M., Vacca, W. D., \& Shuping, Y. S. 2015, in ASP Conf. Ser. 495, Astronomical Data Analysis Software and Systems: XXIV, ed. A. R. Taylor \& E. Rosolowsky (San Francisco. CA: ASP), 355

Cushing, M. C., Vacca, W. D., \& Rayner, J. T. 2004, PASP, 116, 362

Doty, S. D., van Dishoeck, E. F., van der Tak, F. F. S., \& Boonman, A. M. S. 2002, A\&A, 389, 446

Dungee, R., Boogert, A., DeWitt, C. N., et al. 2018, ApJL, submitted

Esplugues, G. B., Viti, S., Goicoechea, J. R., \& Cernicharo, J. 2014, A\&A, 567, A95

Gordon, I. E., Rothman, L. S., Hill, C., et al. 2017, JQSRT, 203, 3

Hatchell, J., Thompson, M. A., Millar, T. J., et al. 2009, A\&A, 504, 853

Indriolo, N., Neufeld., D. A., DeWitt, C. N., Richter, M. J., et al. 2015, ApJL, 802, L14

Jiménez-Serra, I., Zhang, Q., Viti, S., Martín-Pintado, J., \& De-Wit, W.-J. 2012, ApJ, 753, 34

Kaźmierczak-Barthel, M., van der Tak, F. F. S., Helmich, F. P., et al. 2014, A\&A, 567, A53
Keane, J. V., Boonman, A. M. S., Tielens, A. G. G. M., et al. 2001, A\&A, 376, L5

Knez, C., Lacy, J. H., Evans, N. J., van Dishoeck, E. F., \& Richter, M. J. 2013, ApJ, 696, 471

Kurtz, S., Cesaroni, R., Churchwell, E., Hofner, P., \& Walmsley, C. M. 2000, in Protostars and Planets IV, ed. V. Mannings, A. P. Boss, \& S. S. Russell (Tucson, AZ: Univ. Arizona Press), 299

Lacy, J. H., Carr, J. S., Evans, N. J., et al. 1991, ApJ, 376, 556

Lacy, J. H., Knacke, R., Geballe, T. R., \& Tokunaga, A. T. 1994, ApJL, 428, L69

Lacy, J. H., Richter, M. J., Greathouse, T. K., Jaffe, D. T., \& Zhu, Q. 2002, PASP, 114, 153

Li, J., Wang, J., Zhu, Q., Zhang, J., \& Li, D. 2015, ApJ, 802, 40

May, P. W., Pineau des Forêts, G., Flower, D. R., et al. 2000, MNRAS, 318,809

Mitchell, G. F., Curry, C., Maillard, J., \& Allen, M. 1989, ApJ, 341, 1020

Mitchell, G. F., Maillard, J. P., Allen, M., Beer, R., \& Belcourt, K. 1990, ApJ, 363,554

Plambeck, R. L., \& Wright, M. C. H. 1987, ApJL, 317, L101

Rayner, J. T., Tokunaga, A., Jaffe, D., et al. 2016, Proc. SPIE, 9908E, 84R

Richter, M. J., Ennico, K. A., McKelvey, M. E., \& Seifahrt, A. 2010, Proc. SPIE, 7735, 77356Q

Rygl, K. L. J., Brunthaler, A., Sanna, A., et al. 2012, A\&A, 539, 79

Schilke, P., Groesbeck, T. D., Blake, G. A., \& Phillips, T. G. 1997, ApJS, 108, 301

Smith, R. G. 1991, MNRAS, 249, 172

Tercero, B., Cernicharo, J., Pardo, J. R., \& Goioechea, J. R. 2010, A\&A, 517, A96 Tieftrunk, A., Pineau Des Forêts, G., Schilke, P., et al. 1994, A\&A, 289, 579

Turner, B. E., Chan, K., Green, S., \& Lubowich, D. A. 1992, ApJ, 399, 114

van der Tak, F. F. S. 2003, in ASP Conf. Ser. S-221, Star Formation at High Angular Resolution, ed. R. Jayawardhana, M. G. Burton, \& T. L. Bourke (San Francisco, CA: ASP), 59

van der Tak, F. F. S., Boonman, A. M. S., Braakman, R., \& van Dishoeck, E. F. 2003, A\&A, 412, 133

van der Tak, F. F. S., van Dishoeck, E. F., Evans, N. J., \& Bakker, E. J. 1999 ApJ, 522, 991

van Dishoeck, E. F., \& Blake, G. A. 1998, ARA\&A, 36, 317

Villanueva, G. L., Smith, M. D., Protopapa, S., Faggi, S., \& Mandell, A. M. 2018, JQSRT, 217, 86

Viti, S., Collings, M. P., Dever, J. W., McCoustra, M. R. S., \& Williams, D. A. 2004, MNRAS, 354, 1141

Wang, K. S., van der Tak, F. F. S., \& Hogerheijde, M. R. 2012, A\&A, 543, A22

Wilson, T. L., \& Rood, R. T. 1994, ARA\&A, 32, 191

Yang, B., Stancil, P. C., Balakrishnan, N., \& Forrey, R. C. 2010, ApJ, 718, 1062

Young, E. T., Becklin, E. E., Marcum, P. M., et al. 2012, ApJL, 749, L17 